\title{
LINKS OF CLOSED ORBITS OF NONSINGULAR MORSE-SMALE FLOWS
}

\author{
KAZUHIRO SASANO
}

ABSTRaCt. The links which consist of closed orbits of certain nonsingular MorseSmale flows on $S^{3}$ are determined.

Recently various properties of the links of closed orbits of flows have been investigated by several persons (for instance, $[\mathbf{F} 2],[\mathbf{B W}],[\mathbf{M}]$ ). In this paper we determine the link which consists of closed orbits of certain nonsingular Morse-Smale flows on $S^{3}$. (For definitions see below.)

Precisely speaking we prove

THEOREM. Suppose that a nonsingular Morse-Smale flow on $S^{3}$ has a single closed orbit $h_{0}$ of index 2, a single closed orbit $h_{n+1}$ of index 0 , and $n$ closed orbits $h_{1}$, $h_{2}, \ldots, h_{n}$ of index 1 .

(A) If all of the closed orbits of index 1 are untwisted, then the link consisting of all closed orbits is trivial.

(B) If all of the closed orbits of index 1 are twisted, then by reordering $h_{1}, h_{2}, \ldots, h_{n}$ appropriately, we find $k$ such that

(a) $h_{k}$ and $h_{k+1}$ make the Hopf link,

(b) for any $i<k, h_{i}$ is a $\left(2, p_{i}\right)$-cable of $h_{i+1}$, and

(c) for any $j>k, h_{j+1}$ is a $\left(2, q_{j}\right)$-cable of $h_{j}$, where $p_{i}$ and $q_{j}$ are arbitrary odd integers. (See Figure 1.)

COROllary. Suppose that a nonsingular Morse-Smale flow on $S^{3}$ has three closed orbits. Then there exists just one closed orbit of index $i$ for each $i(0 \leqslant i \leqslant 2)$, and the closed orbits make either the trivial link or the link as in Figure 2, where $h$ is the orbit of index 1 and $L_{1}$ is a $(2, p)$-cable of $h(p=$ odd $)$, and one of $L_{1}$ and $L_{2}$ is of index 0 and the other is of index 2.

The author is very grateful to Professors Itiro Tamura, Shigenori Matsumoto and Koich Yano for their advice and encouragement, Professor Nagayoshi Iwahori for his advice on Algebra, and Noriko Maruyama for her encouragement. Finally the author thanks the referee for his useful suggestions.

Received by the editors June 28, 1982.

1980 Mathematics Subject Classification. Primary 58F09; Secondary 57M25.

Key words and phrases. Link, nonsingular Morse-Smale flow. 


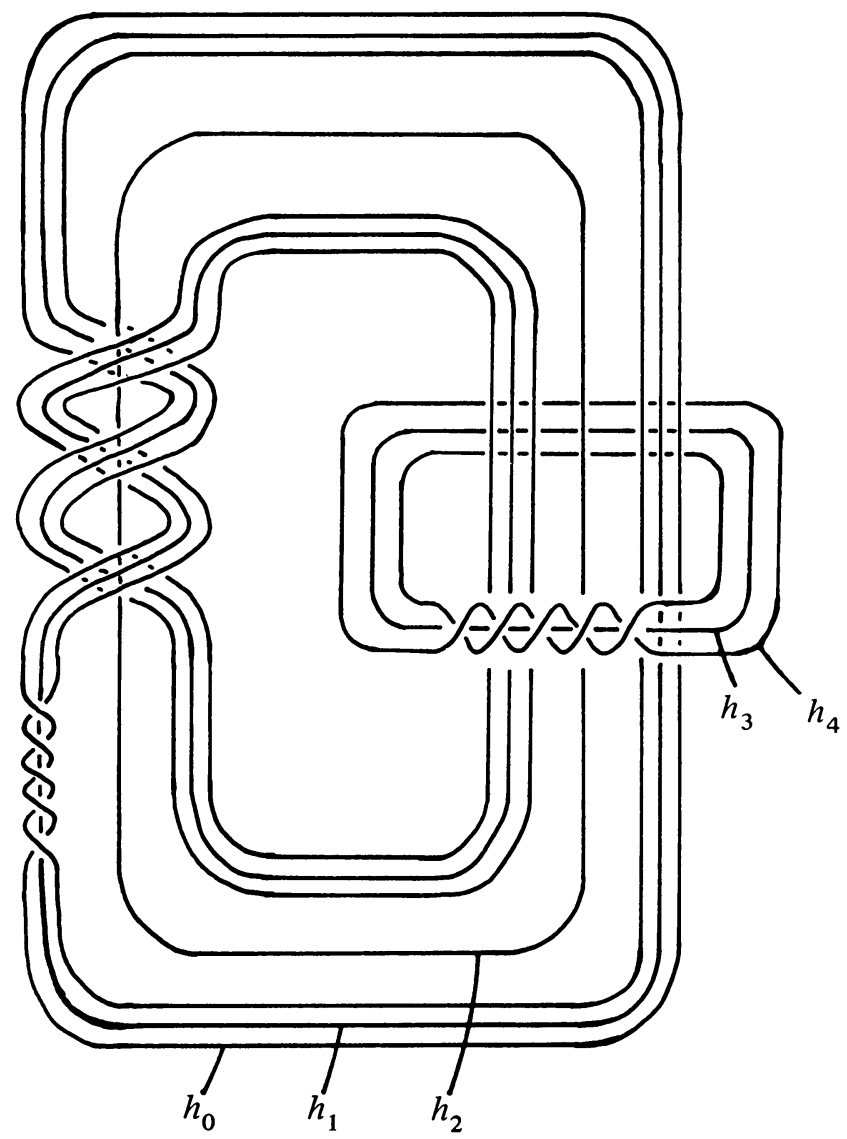

(This figure shows the case $n=3, k=2$.)

FIGLRE 1

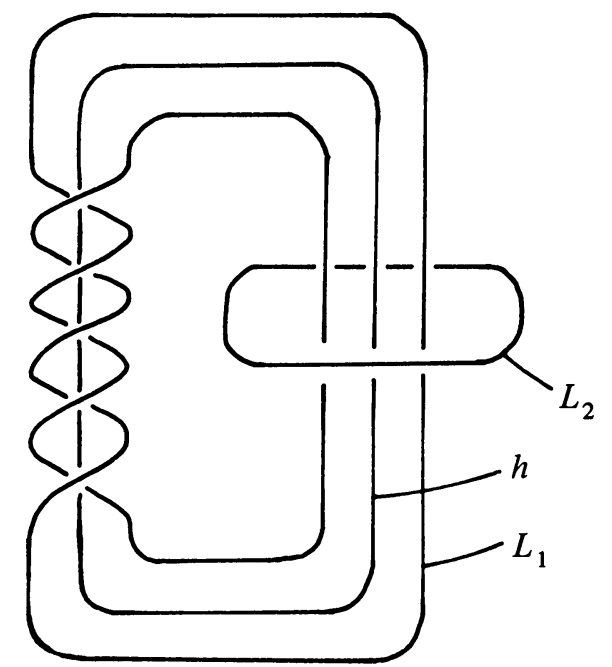

FIGURE 2 
1. Definitions. In this section we recall fundamental definitions and properties briefly. For further details see [F3], [M], [Sm]. On the low-dimensional topology see $[\mathbf{R}]$.

A nonsingular Morse-Smale flow (or an NMS flow for short) on a manifold $M^{n}$ is a flow without fixed points which satisfies the following conditions:

(1) The nonwandering set consists entirely of a finite number of closed orbits.

(2) The Poincare map for each closed orbit is hyperbolic.

(3) If $c$ and $c^{\prime}$ are closed orbits, then the stable manifold of $c$ and the unstable manifold of $c^{\prime}$ intersect transversely.

Then the dimension of the unstable bundle of a closed orbit $c$ is called the index of c. A closed orbit is called untwisted if its unstable bundle is orientable. Otherwise it is called twisted.

Associated to an NMS flow, we can consider a round handle decomposition of $M^{n}$.

Definition 1.1. (a) Let $X^{n}, Y^{n}$ be manifolds. $X^{n}$ is obtained from $Y^{n}$ by attaching a round $k$-handle if

(1) there are disk bundles $E_{s}^{k}$ and $E_{u}^{n-k-1}$ over $S^{1}$, and

(2) an embedding $\theta: \partial E_{s}^{k} \times E_{u}^{n-k-1} \rightarrow \partial Y^{n}$ such that

$$
X^{n} \approx Y_{\theta}^{n} \cup_{\theta}\left(E_{s}^{k} \oplus E_{u}^{n-k-1}\right) .
$$

(b) The total space of $E_{s}^{k} \oplus E_{u}^{n-k-1}$ is called a round $k$-handle.

(c) A round handle decomposition for $X^{n}$ is a filtration $X_{0} \subset X_{1} \subset X_{2} \subset \cdots \subset X_{k}$ $=X$, where each $X_{i}$ is obtained from $X_{i-1}$ by attaching a round handle.

D. Asimov and J. Morgan connected an NMS flow with a round handle decomposition as follows.

Proposition 1.2 [A], [M]. If a manifold $M$ has an NMS flow, then $M$ has a round handle decomposition whose core circles are the closed orbits of the flow. Conversely, if $M$ has a round handle decomposition, then $M$ has an NMS flow whose closed orbits are the core circles of round handles.

A round handle is called untwisted if its core circle is an untwisted closed orbit. Otherwise it is called twisted.

In the rest of this paper we consider an NMS flow on $S^{3}$. Then a round 1-handle $H$ is of the form $H=E_{s}^{1} \oplus E_{u}^{1}$ and the part $\partial E_{s}^{1} \times E_{u}^{1}$ of $\partial H$ is two copies of annuli if $H$ is untwisted, or an annulus if $H$ is twisted. Each annulus is mapped to a small tubular neighborhood of a circle on the boundary surface of a 3-manifold. Such a circle is called the attaching circle of $H$.

2. Preliminaries. In this section we give preliminary lemmas which are necessary to prove our main theorem. Let an NMS flow be given on $S^{3}$.

LEMMA 2.1. Let $U$ be a solid torus in $S^{3}$ such that the flow is outwardly transverse to the boundary $\partial U$ of $U$. Let $H$ be an untwisted round 1-handle with core $h$, which is attached to $U$. Then one of the following holds.

(A) The boundary of the resulting manifold $U \cup H$ is the disjoint union of two tori.

(B) $U \cup H$ is a solid torus, in which $U$ and h are put trivially (see Figure 2.1). 


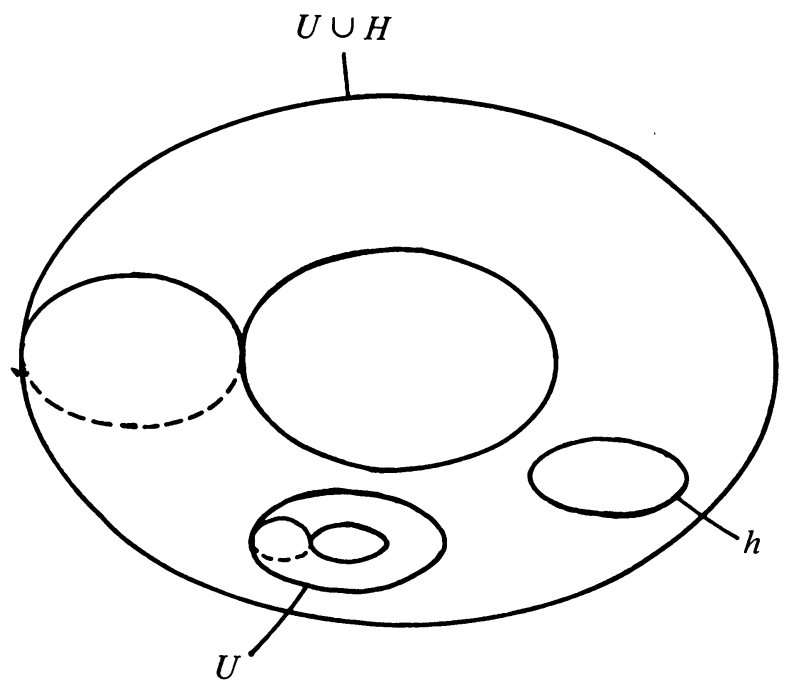

FIGURE 2.1

Proof. Let $K_{1}$ and $K_{2}$ be attaching circles of $H$ on $U$. If $\left[K_{1}\right]=\left[K_{2}\right]=0$ in $H_{1}(\partial U)$ then four cases as in Figure 2.2 occur. In the case (a), the boundary of $U \cup H$ contains $S^{2}$ as a connected component. $S^{2}$ bounds a 3 -ball and the flow is transverse to $S^{2}$. But such a flow in a 3-ball has a fixed point. This contradicts our assumption. In the case (b) or (d), the Klein bottle is embedded in $S^{3}$. This is a contradiction. In the case (c), we have (A) in our lemma. If $\left[K_{1}\right]=0$ and $\left[K_{2}\right]=$ $a\left[m_{u}\right]+b\left[l_{u}\right] \neq 0$, where $m_{u}$ and $l_{u}$ are the meridian and the longitude of $U$, then $a=\operatorname{lk}\left(\left[K_{2}\right],[u]\right)=\operatorname{lk}\left(\left[K_{1}\right],[u]\right)=0$, where $u$ is the core of $U$ and $\operatorname{lk}($, , denotes the linking number. Thus $b= \pm 1$, and we obtain (B). If $\left[K_{1}\right]=\left[K_{2}\right] \neq 0$, we have (A). This completes the proof.

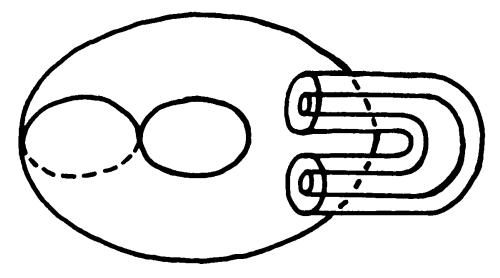

(a)

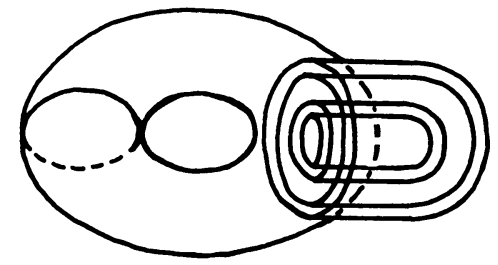

(c)

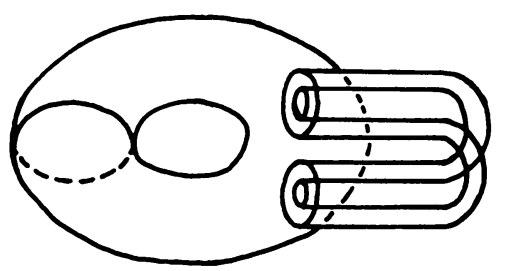

(b)

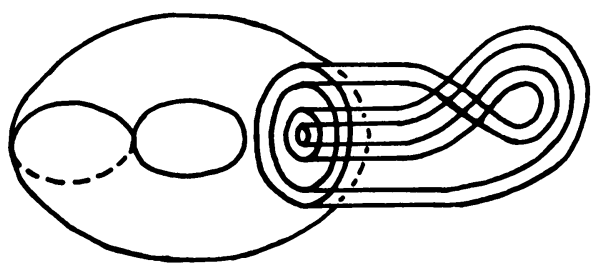

(d)

FigURE 2.2 


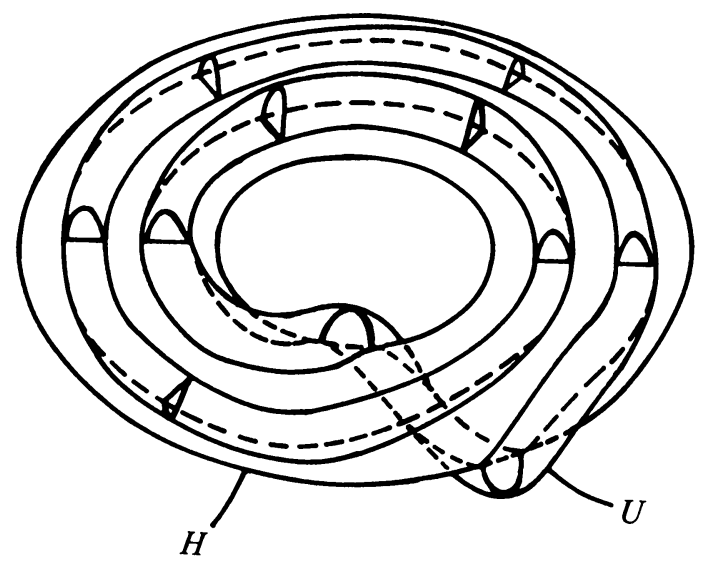

(A)

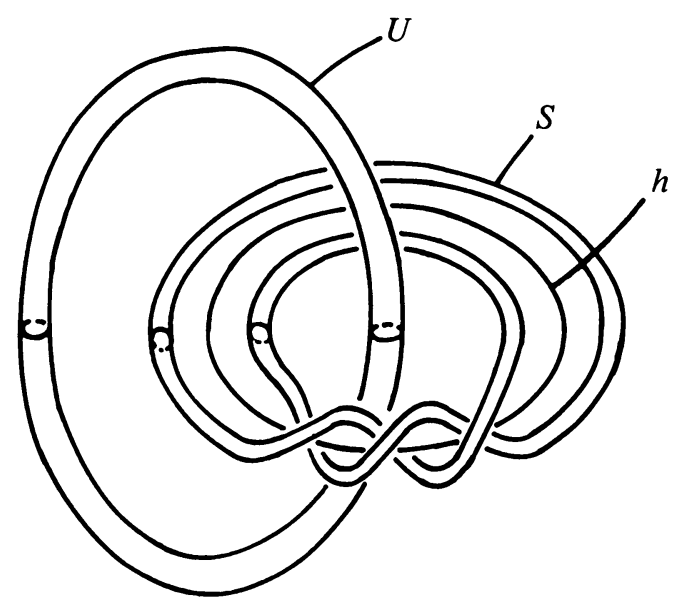

(B)

Figure 2.3

LEMMA 2.2. Let $U$ be a solid torus in $S^{3}$ such that the flow is outwardly transverse to the boundary of $U$. Let $H$ be a twisted round 1-handle with core $h$, which is attached to $U$. Let $S=S^{3}-(U \cup H)$. Then $U \cup H$ is one of the following.

(A) $A$ solid torus.

(B) The exterior of a $(2, p)$-torus knot, where $p$ is an arbitrary odd integer. In this case $S$ is the tubular neighborhood of the knot, and $U$ is an unknotted solid torus (see Figure 2.3).

Proof. Let $K$ be the attaching circle of $H$ on $\partial U$. Let $m_{u}$ and $l_{u}$ be the meridian and the longitude of $U$. If $[K]=0$ or $[K]=\left[m_{u}\right]$ in $H_{1}(\partial U)$, then the projective plane $P^{2}$ is embedded in $S^{3}$. This is a contradiction. Suppose $[K]=a\left[m_{u}\right]+b\left[l_{u}\right]$, where $b \neq 0$. We may assume $b>0$ and $-b / 2<a<b / 2$. If $b=1$, then $a=0$ and $U \cup H$ is a solid torus. This gives (A). Assume $b>1$. By the solid torus theorem 
[R, p. 107], the boundary torus $\partial(U \cup H) \approx T^{2}$ bounds a solid torus on at least one side. But

$$
\pi_{1}(U \cup H)=\left\langle h, u ; h^{2} u^{h}=1\right\rangle \neq \mathbf{Z},
$$

where $h$ and $u$ are the generator of the fundamental group represented by the orbit $h$ and the core $u$ of $U$. Thus $S$ is a solid torus and $U \cup H$ is a knot exterior. Hence $H_{1}(U \cup H)=\mathbf{Z}$. So $b$ is odd. The fundamental group of the boundary $\partial(U \cup H)$ is generated by $u^{b}$ and $h u^{x}$, where $x$ is the integer such that $a x \equiv-1(\bmod b)$ and $0<x<b$. Let $\psi: \partial(U \cup H) \rightarrow \partial S$ be the inverse of the attaching map of $S$ to $U \cup H$, and assume that the induced map $\psi_{*}: H_{1}(\partial(U \cup H)) \rightarrow H_{1}(\partial S)$ is given by

$$
\psi_{*}\left(\left[u^{h}\right]\right)=q\left[m_{s}\right]+r\left[l_{s}\right] \text { and } \psi_{*}\left(\left[h u^{x}\right]\right)=s\left[m_{s}\right]+t\left[l_{s}\right] \text {, }
$$

where $m_{s}$ are $l_{s}$ are the meridian and the longitude of $S$ and $q t-r s= \pm 1$. Then $\psi_{*}^{-1}\left(\left[m_{s}\right]\right)= \pm\left(t\left[u^{b}\right]-r\left[h u^{x}\right]\right)$. Hence we have

$$
\pi_{1}((U \cup H) \cup S)=\left\langle h, u ; h^{2} u^{b}=1, u^{b t}\left(h u^{x}\right)^{-r}=1\right\rangle .
$$

We denote this group by $G$. Since $(U \cup H) \cup S=S^{3}, G$ should be a trivial group. We prepare

LEMMA 2.3. $G$ is trivial if and only if $t=0, r= \pm 1$, and $x=(b \pm 1) / 2$ (consequently $a=\mp 2$ ).

Proof of Lemma 2.3. Let

$$
\tilde{G}=\left\langle\tilde{h}, \tilde{u} ; \tilde{h}^{2}=\tilde{u}^{b}=\left(\tilde{h} \tilde{u}^{x}\right)^{r}=1\right\rangle .
$$

Note that $\tilde{G}$ is a surjective image of $G$, and $G=\{1\}$ implies $\tilde{G}=\{1\}$. By putting $\tilde{v}=\tilde{u}^{x}$ and $\tilde{w}=(\tilde{h} \tilde{v})^{-1}, \tilde{G}$ is rewritten as

$$
\tilde{G}=\left\langle\tilde{h}, \tilde{v}, \tilde{w} ; \tilde{h}^{2}=\tilde{v}^{b}=\tilde{w}^{r}=\tilde{h} \tilde{v} \tilde{w}=1\right\rangle .
$$

By a famous theorem of $\mathrm{H}$. Coxeter $[\mathbf{C M}], \tilde{G}=\{1\}$ if and only if $r= \pm 1$. Thus $G=\{1\}$ implies $r= \pm 1$. Conversely, assume $r= \pm 1$. Then $G=\left\langle u ; u^{2(-x \pm t b)+b}=\right.$ $1\rangle$. Hence it is necessary that $t=0$ and $x=(b \pm 1) / 2$. This completes the proof.

We continue the proof of Lemma 2.2. By Lemma 2.3, $r= \pm 1$ and $\psi_{*}\left(\left[u^{b}\right]\right)=$ $q\left[m_{s}\right] \pm\left[l_{s}\right]$. Under an appropriate diffeomorphism of $S$, we may assume $\psi_{*}\left(\left[u^{b}\right]\right)$ $=\left[l_{s}\right]$. Since the core of the part of $\partial H$ attached to $\partial S$ (with respect to the flow with the reversed direction) represents $h^{2}$ in $\pi_{1}(H)$, the attaching circle of $H$ on $\partial S$ is the longitude of $S$. Hence this case reduces to the case of $b=1$, by reversing the direction of the flow. So $H \cup S$ is a solid torus and $U$ is an unknotted torus. This completes the proof.

\section{Proof of Theorem and Corollary.}

Proof of Theorem. Let $h_{i}$ be as in the Theorem and $H_{i}$ be a round handle with core $h_{i}$. By reordering $h_{i}$ 's if necessary, we may assume that $H_{i+1}$ is attached to $\cup_{0 \leqslant j \leqslant i} H_{j}$. Let $H(i)=\cup_{0 \leqslant j \leqslant i} H_{j}(0 \leqslant i \leqslant n+1)$.

(A) Assume that all of $h_{1}, h_{2}, \ldots, h_{n}$ are untwisted. We prove, by induction on $i$, that $H(i)$ is a solid torus for any $i=0,1, \ldots, n$. For $i=0$, it is trivial. Assume that it 
is proved for $i<n$. Applying Lemma 2.1 to $U=H(i)$ and $H=H_{i+1}$, we have two cases. If (A) of Lemma 2.1 occurs, then the boundary of $H(i+1)$ is the disjoint union of two tori and the flow is outwardly transverse to these tori. To make $S^{3}$ which is without boundary, we must connect these tori by $H_{i+2} \cup H_{i+3}$ $\cup \cdots \cup H_{n+1}$. Hence we can choose a simple closed curve which intersects an embedded torus (each component of the boundary of $H(i+1)$ ) in $S^{3}$ just one time. This leads to a contradiction. Thus $H(i+1)$ is a solid torus, and the induction is completed. Note that $H(i)$ and $h_{i+1}$ are put trivially in $H(i+1)$. Thus it is proved inductively that $h_{0}, h_{1}, \ldots, h_{n}$ are put trivially in the solid torus $H(n)$. Attaching $H_{n+1}$ to $H(n)$ so that $H(n) \cup H_{n+1}=S^{3}$, we can prove (A) of the Theorem immediately.

(B) Assume that all of $h_{1}, h_{2}, \ldots, h_{n}$ are twisted. Let $k$ be the maximum number such that $H(i)$ is a solid torus for every $i=0,1, \ldots, k$. If $k=n$ then (B) of the Theorem is immediate. We assume $k<n$. By reversing the direction of the flow, we may regard that $H_{i}$ is attached to $H_{i+1} \cup \cdots \cup H_{n+1}$. Let $S(i)=\cup_{i \leqslant j \leqslant n+1} H_{j}$. Applying Lemma 2.2 to $U=H(k)$ and $H=H_{k+1}$, we have that $S(k+2)=S^{3}-$ $\left(H(k) \cup H_{k+1}\right)$ is a solid torus. We will prove that $S(i)$ is a solid torus for $i=k+1, k+2, \ldots, n+1$. Let $l$ be the minimum number so that $S(i)$ is a solid torus for every $i=l, l+1, \ldots, n+1$. We will show $l=k+1$. Assume $l=k+2$. Applying Lemma 2.2 to $U=H(k), H=H_{k+1}, S=S(k+2)$, since $S(k+2)$ is a solid torus and $H(k+1)=H(k) \cup H_{k+1}$ is not a solid torus, $S(k+1)=S(k+2)$ $\cup H_{k+1}$ is also a solid torus. This contradicts the choice of $l$. So $l \neq k+2$. Assume $l>k+2$. Let $s$ be the core of the solid torus $S(l)$. Then

$$
\pi_{1}(S(l))=\langle s\rangle=\mathbf{Z} \text { and } \pi_{1}(S(l-1))=\left\langle h_{l-1}, s ; h_{l-1}^{2} s^{h}=1\right\rangle .
$$

where $b$ is an odd integer greater than or equal to 3 . Inductively we have

$$
\begin{aligned}
& \pi_{1}(S(k+2)) \\
& \quad=\left\langle h_{k+2}, h_{k+3}, \ldots, h_{l-1}, s ; h_{l-1}^{2} s^{b}=1, h_{l-2}^{2}=r_{l-2}, \ldots, h_{k+2}^{2}=r_{k+2}\right\rangle,
\end{aligned}
$$

where $r_{j}$ is a word in $h_{j+1}, h_{j+2}, \ldots, h_{l-1}, s$. This group contains $\left\langle h_{l-1}, s ; h_{l-1}^{2} s^{b}=1\right\rangle$ as a subgroup. Hence this group is not $\mathbf{Z}$. This contradicts the fact that $S(k+2)$ is a solid torus. Hence $l=k+1$. Now the proof of $(\mathrm{B})$ of the Theorem is easy.

The proof of the Theorem is completed.

Proof of Corollary. J. Franks proved that the number $A_{i}$ of untwisted closed orbits of index $i$ of any NMS flow on $S^{3}$ satisfies that $A_{0} \geqslant 1, A_{2} \geqslant 1$, and $A_{1} \geqslant \max \left(A_{0}-1, A_{2}-1\right)$. Thus there exists just one closed orbit of index $i$ for each $i$. The rest of the Corollary is immediately proved by the Theorem.

4. Concluding remarks. Our last aim is the complete classification on links of closed orbits of NMS flows. We give some results for our aim.

(1) The author obtained the complete classification of links of closed orbits of any NMS flow with at most 5 closed orbits on $S^{3}$. For the result, consult [Sa].

(2) K. Yano [Y] proved that any NMS flow on $S^{3}$ has at least two unknotted closed orbits. 


\section{REFERENCES}

[A] D. Asimov, Round handles and non-singular Morse-Smale flows, Ann. of Math. (2) 102 (1975). 55-64.

[BW] J. Birman and R. F. Williams, Knotted periodic orbits in dinamical systems. I, II, preprint.

[CM] H. S. M. Coxeter and W. O. J. Moser, Generators and relations for discrete groups, Springer-Verlag, Berlin and New York, 1957 (2nd ed., 1965).

[F1] J. Franks, The periodic structure of non-singular Morse-Smale flows, Comment. Math. Helv. 53 (1978), 279-294.

[F2] _. Knots, links, and symbolic dinamics, Ann. of Math. (2) 113 (1981), 529-552.

[F3] Homologi and dinamical sistems, preprint.

[M] J. Morgan, Non-singular Morse-Smale flows on 3-dimensional manifolds, Topology 18 (1978), 41-53.

[R] D. Rolfsen, Knots and links, Publish or Perish, Berkeley, Calif., 1976.

[Sm] S. Smale, Differentiable dinamical sy'stems, Bull. Amer. Math. Soc. 73 (1967), 747-817.

[Sa] K. Sasano, Links in some simple flows (in preparation).

[Y] K. Yano, Private communication, May 1982.

Department of Mathematics, Faculty of SCience, University of Tokyo, Hongo, Tokyo 113, JAPAN 